\title{
Tests for Supernova Explosion Models: from Light Curves to X-ray Emission of Supernova Remnants
}

\author{
Elena Sorokina ${ }^{1}$ and Sergey Blinnikov ${ }^{2}$ \\ 1 SAI, Universitetskij pr. 13, 119992 Moscow, Russia; \\ sorokina@sai.msu.su \\ 2 ITEP, B. Cheremushkinskaya 25, 117218 Moscow, Russia; \\ blinn@sai.msu.su
}

Summary. The successful theoretical supernova explosion models should be able to explain any features of the emission from supernovae at any evolutionary stage. We check several models from two different points of view. With the multi-frequency radiation hydro code STELLA we calculate gamma-ray, bolometric and broad-band UBVI light curves. Then we use the same models to calculate the emission from young supernova remnants. Here we present new plots for gamma-ray luminosity from several SN Ia models and recomputations of bolometric and UBVRI light curves of model 13C for SN 1993J.

\section{Introduction}

So far, there exist many explosion models proposed by theorists for different types of supernovae, but still there are no definite criteria to decide which of the models are realized in nature. Only a few parameters, such as kinetic energy and total ${ }^{56} \mathrm{Ni}$ production, can be derived directly from the modeling of the explosion and compared with the observational values. The subsequent evolution of the exploded star gives us much more possibilities to compare models and to decide which one fits observations better.

The first possibility is modeling of gamma-ray luminosity of supernovae. The comparison with the observational values allows to define the total mass of radioactive isotopes, to judge on the composition of the outer layers where gamma-rays are absorbed and thermalized, and also to check the approximations of gamma-ray opacity. During the first months after an explosion one can examine a theoretical model by calculating bolometric and monochromatic light curves and spectra, and comparing them with observations. Later on, gas in the ejecta cools down and becomes almost unobservable. The next opportunity to analyze the ejecta is on the stage of a young supernova remnant (SNR), when noticeable amount of circumstellar gas is swept up. Then a reverse shock forms, goes inwards the ejecta and illuminates them once again.

The successful theoretical supernova explosion models should be able to explain any feature of the emission from supernovae at any evolutionary 
Table 1. Parameters of SN Ia models

\begin{tabular}{llllll}
\hline Model & DD4 & W7 & LA4 & WD065 MR \\
\hline$M_{\mathrm{WD}}{ }^{\mathrm{a}}$ & 1.3861 & 1.3775 & 0.8678 & 0.6500 & 1.4 \\
$M_{56 \mathrm{Ni}}{ }^{\mathrm{a}}$ & 0.63 & 0.60 & 0.47 & 0.05 & 0.42 \\
$E_{51}{ }^{\mathrm{b}}$ & 1.23 & 1.20 & 1.15 & 0.56 & 0.46 \\
\hline${ }^{\mathrm{a}}$ in $M_{\odot}$ & & & & \\
${ }^{b}$ in $10^{51}$ ergs s$^{-1}$ & & &
\end{tabular}

stage. Different combinations of codes we have in our group allows us to compute the evolution of models, and therefore test them, on several stages. For calculations of broad-band optical and bolometric light curves we use the multi-frequency radiation hydro code STELLA. At the young remnant stage, while gas is transparent, we calculate the evolution and X-ray emission by combination of hydro part of STELLA with the non-equilibrium ionization code which is based on the original algorithm by Peter Lundqvist.

\section{SN Ia Models}

We choose five models of Type Ia supernovae which are shown in Table 1. W7 [8] and DD4 [14] are more or less similar classical 1D Chandrasekhar mass models. LA4 [7] and WD065 [11] are sub-Chandrasekhar mass ones. (See original works for details.) The last model was computed a couple of years ago in Garching, in the Max-Planck-Institute group [9]. It is originally $3 \mathrm{D}$. We average it over $4 \pi$ for our calculation, since we use only $1 \mathrm{D}$ codes at the moment. The main feature of the model is that it is very well mixed, while energetics and the amount of $\mathrm{Ni}^{56}$ are lower than in other Chandrasekhar mass models. In our calculations we compare two versions of the model with the same hydro part, but different element distributions.

\section{Results}

\subsection{SNe Ia}

Now there appears more and more possibilities to obtain gamma-ray light curves using modern gamma-ray space observatories, like COMPTEL [5]. Here we just compare what one would observe after the explosion of the models we have discussed.

In Fig. 1 we compare the light curves in gamma-rays produced by different models. We have chosen W7 as a representative case of Chandrasekhar-mass models. The light curves in gamma-rays are more or less similar for all of them. The luminosity of the MR model is lower due to smaller amount of 

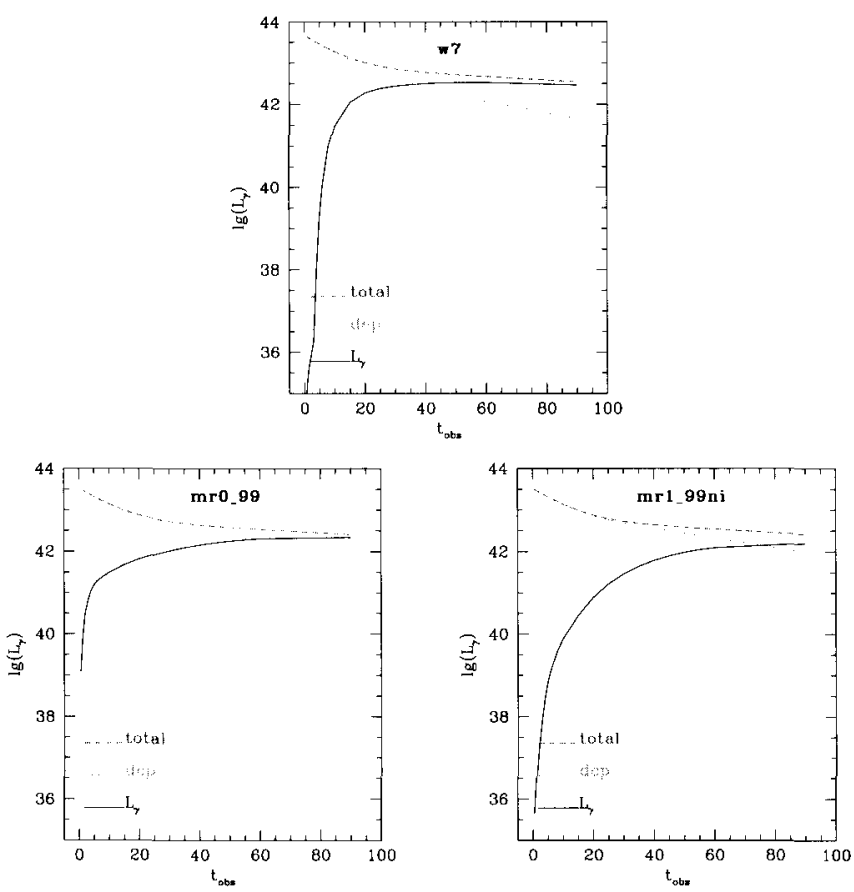

Fig. 1. Gamma-ray production by $\mathrm{Ni}$ and Co decays (dashes), gamma-ray power deposited into heating (dots), and total gamma-ray luminosity of SN (solid) versus time in days for different explosion models: W7 (top panel), MR model averaged over $4 \pi$ (bottom left), and MR model averaged over an opening angle of $14^{\circ}$ with the same mass of ${ }^{56} \mathrm{Ni}$, which is situated mostly near the center (bottom right).

${ }^{56} \mathrm{Ni}$, and it rises slower since it is less energetic, so $\gamma$-photons are locked inside the ejecta for longer time. One can see even smaller number of $\gamma$-photons during the first weeks after the explosion in the model similar to MR but less mixed, with ${ }^{56} \mathrm{Ni}$ residing mostly near the center. The maximum luminosity in gamma-rays is still the same as in original MR, since we preserve the total amount of ${ }^{56} \mathrm{Ni}$.

Therefore, the light curve in gamma-rays during the first months can be divided into two epochs, that represent different physical parameters of the explosion: the first $40-60$ days and the following evolution. From the observations during the first period one can judge on the combination of explosion energy and the distribution of radioactive stuff over the ejecta, while the second period tells us mostly about total production of radioactive elements at the explosion. Since currently the observations can only provide us with fluxes integrated over several days (or even weeks), it seems expedient to make two different sets of observations: during the first two months after the 

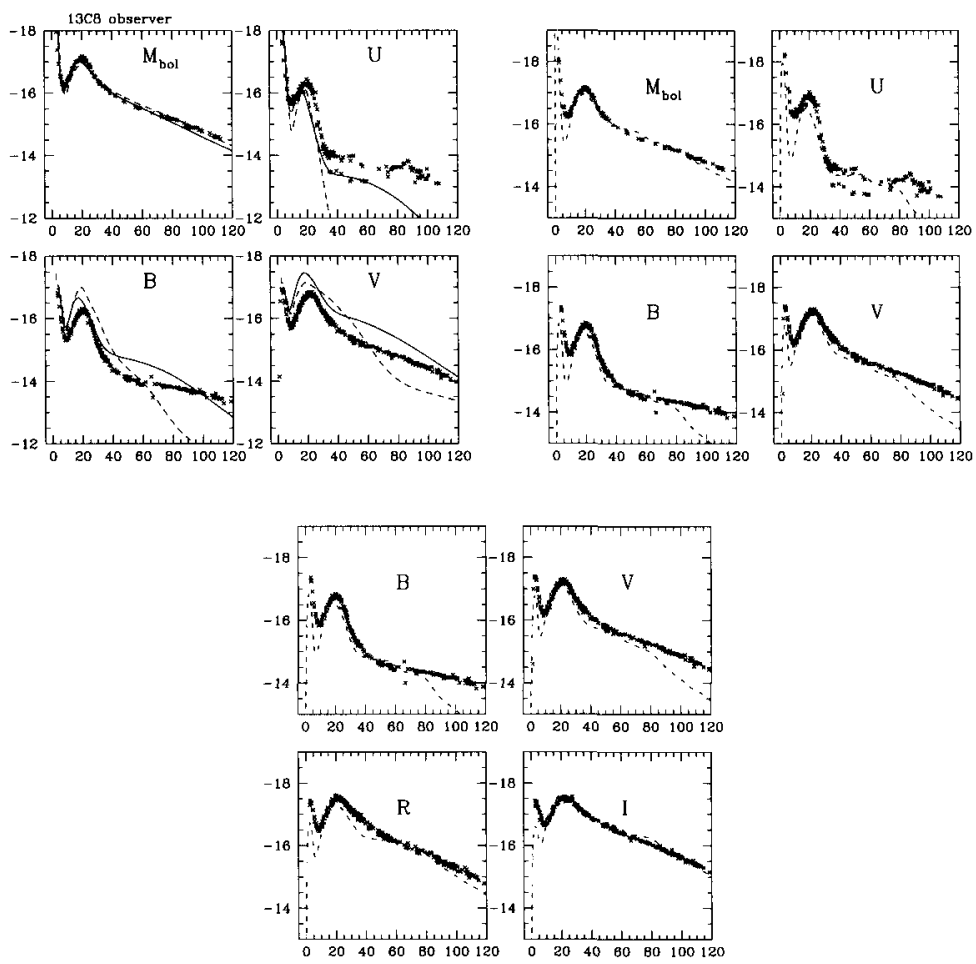

Fig. 2. Bolometric and UBVRI light curves for SN 1993J. Top left panel: The best model 13C8 from [2] (solid line - calculations with STELLA, dashes - with EDDINGTON). Top right and bottom panels: Our recalculation of the same model with a new version of STELLA. Observational data are taken from [10] and shown by crosses. The value of reddening is taken to be $E(B-V)=0.08$.

explosion, and after that, in order to distinguish between different parameters of explosion, and to test explosion models.

We have described our modeling of bolometric and UBVRI light curves and calculations of X-ray emission of young SNRs in previous work. For details one can see, e.g., $[3,4,6]$. We just mention here that a bit less energetic and more mixed models, like MR, seem to us more preferable both for SN light curve and for SNR X-ray emission. At the latter point, our results seem to contradict the conclusions by [1], that iron lines from well mixed models at the SNR stage are too strong, while less mixed models give a better agreement with observations of Tycho. Still we trust in our results more, since we take into account the ionization energy in the equation of state, which is comparable to the thermal energy. Electron thermal conductivity is also included into our calculations, and it smoothes very much the temperature profile between forward and reverse shocks. Both these effects are neglected 
in the code by [1], though they are able to change the emitted spectrum appreciably. The code by [1] takes into account possible difference between electron and ion temperature using the standard Coulomb collision equation, while we treat this effect parametrically. This can also lead to differences in our results. The work on improving physics and making our code more self-consistent is in progress.

\section{$3.2 \mathrm{SN}$ 1993J}

In order to pay attention to the $\mathrm{SN}$ entitling this conference, we have recalculated its light curve. We use the same model $13 \mathrm{C}$ from [13] which was investigated a few years ago in [2]. It is one of the best models for SN 1993J, and we just wanted to check if the new version of our code STELLA, with improved and renovated physics, still produces the light curve that fits observations well.

The results of previous and current calculations are compared in Fig. 2. The main improvement in the code is a new approach for expansion opacities [12]. They become much more complicated, and, most probably, the bumps on the new light curves are the results of this improvement. But sometimes (for instance, in the $\mathrm{U}$ band) these bumps seem to fit the observations even better than it was in the old version. $R$ and $I$ bands are calculated with STELLA for the first time, and they look perfect.

There is a discussion in the literature on the estimates of the reddening to SN 1993J ([10] and references therein). In the plots in Fig. 2 we assume $E(B-V)=0.08$. With larger value, the observational curves become higher than the modeled ones. So the new version of STELLA confirms that the model 13C corresponds to the explosion of SN 1993J very well, but in the case of low reddening.

Acknowledgement. ES is grateful to the organizers of the meeting for their warm hospitality and support. The work is supported by RFBR through grants 02-02$16500,03-02-06770$, and 03-02-26598.

\section{References}

1. C. Badenes, E. Bravo, K.J. Borkowski, I. Domínguez: Astrophys. J. 593, 358 (2003)

2. S.I. Blinnikov et al. : Astrophys. J. 496, 454 (1998)

3. S. Blinnikov, E. Sorokina: Astron. Astrophys. 356, L30 (2000)

4. S. Blinnikov, E. Sorokina: "Type Ia Supernova Models: Latest Developments." In: Hunting the Cosmological Parameters, ed. D. Barbossa et al. (Kluwer: Dordrecht, 2003)

5. R. Georgii, S. Plüschke, R. Diehl et al: Astron. Astrophys. 394, 517 (2002) 
6. D.I. Kosenko, E.I.Sorokina, S.I. Blinnikov, P. Lundqvist: "X-ray Emission of Young SN Ia Remnants as a Probe for an Explosion Model." In: Proc. of 34 th Scientific Assembly of COSPAR, (ASR: 2003)

7. E. Livne, D. Arnett: Astrophys. J. 452, 62 (1995)

8. K. Nomoto, F.-K. Thielemann, K. Yokoi: Astrophys. J. 286, 644 (1984)

9. M. Reinecke, W. Hillebrandt, J.C. Niemeyer: Astron. Astrophys. 386, $936(2002)$

10. M.W. Richmond et al. : Astron. J. 107, 1022 (1994)

11. P. Ruiz-Lapuente et al. : Nature 365, 728 (1993)

12. E.I. Sorokina, S.I. Blinnikov: "Energy exchange inside SN ejecta and light curves of SNe Ia." In: Nuclear Astrophysics, MPA/P13, ed. W. Hillebrandt, E. Müller (Garching: 2002) pp. 57-62

13. S.E. Woosley, R.G. Eastman, T.A. Weaver, P.A. Pinto: Astrophys. J. 429, $300(1994)$

14. S.E. Woosley, T.A. Weaver: "Massive Stars, Supernovae, and Nucleosynthesis." In: Supernovae, ed. R. Bludman et al. (Elsevier Sci. Pub.: Amsterdam, 1994) pp. $63-154$ 